\title{
MicroRNA-27a-3p regulates epithelial to mesenchymal transition via targeting YAP1 in oral squamous cell carcinoma cells
}

\author{
GUANG ZENG ${ }^{1}$, WENXING XUN ${ }^{1}$, KEWEN WEI $^{1}$, YONGJIN YANG ${ }^{2}$ and HUAN SHEN ${ }^{2}$ \\ ${ }^{1}$ Department of Plastic and Burn Surgery, Tangdu Hospital, The Fourth Military Medical University, Xi'an, Shaanxi 710038; \\ ${ }^{2}$ Department of Stomatology, The General Hospital of the Second Artillery Corps of Chinese PLA, Beijing 100088, P.R. China
}

Received January 27, 2016; Accepted May 30, 2016

DOI: $10.3892 /$ or.2016.4916

\begin{abstract}
MicroRNAs (miRNAs) are small non-coding RNAs frequently dysregulated in human malignancies. Here, we profiled isolated cells from freshly resected tumors from oral squamous cell carcinoma (OSCC) patients and OSCC cell lines using a SYBR Green-based qPCR miRNA array to identify the expression change of the miRNAs. Based on the microarray data and clincopathological factor analysis of 50 OSCC patients related to these miRNAs, miR-27a-3p was selected as a putative miRNA which might play important role in OSCC progression. By bioinformatics analysis and dual-luciferase reporter assay, we found that YAP1 (Yes-associated protein-1) was a direct target gene of miR$27 \mathrm{a}-3 \mathrm{p}$. Intriguingly, increased expression of miR-27a-3p could significantly decrease the expression level of YAP1 as well as several epithelial to mesenchymal transition (EMT)related molecules in OSCC cell lines, including Twist and Snail. Then, follow-up studies revealed that miR-27a-3p expression was able to downregulate the EMT-related molecules effectively, which might be involved in the regulation of Sox 2 via the YAP1-OCT4-Sox2 signaling axis. In summary, this study found that miR-27a-3p could inhibit the YAP1 directly by post-transcriptionally silencing and potentially suppress EMT process, suggesting that miR-27a-3p might play pivotal roles in effectively manipulating the invasion and metastasis in oral squamous cell carcinoma cells through the EMT inhibition.
\end{abstract}

\section{Introduction}

Oral squamous cell carcinoma (OSCC) is the 6th most frequent malignant neoplasm and remains a lethal disease for more than $50 \%$ of the patients annually. OSCC patients die

Correspondence to: Dr Huan Shen, Department of Stomatology, The General Hospital of the Second Artillery Corps of Chinese PLA, Beijing 100088, P.R. China

E-mail: uyhqyi@163.com

Key words: miR-27a-3p, Yes-associated protein-1, OCT4, epithelial to mesenchymal transition, oral squamous cell carcinoma of tumor metastasis and recurrence due to its strong invasion potential (1). MicroRNAs (miRNAs) are small non-coding RNAs which are frequently dysregulated in human malignancies (2). Recently, miRNAs have emerged as fundamental regulators in gene expression through silencing gene expression at the post-transcriptional and translational levels. In this study, microarray analysis of miRNA expression change in the OSCC patient specimens and OSCC cell lines was firstly identified. Through the analysis of the multiple experimental data, miRNAs that displayed $>3$-fold changes in OSCC patients and cell lines were recorded. Then, miR-27a-3p was selected as the most potential gene which might be involved in the occurrence and development progress of OSCC when combined with the clinical prognosis and $\mathrm{T}$ stage in the statistical analysis. However, the underlying mechanism of OSCC related to miR-27a-3p still remains largely unknown.

In this study, we aimed to reveal the biological function of miR-27a-3p related to OSCC, especially in the tumor invasion. Through Target prediction algorithms (3) and dual-luciferase reporter assays, we found that YAP1 (Yes-associated protein-1) was a direct target gene of miR27a-3p. Consistently, the expression of active YAP1 was negatively correlated with that of miR-27a-3p in OSCC cell lines. YAP1, a transcriptional co-activator, is the oncogenic component of the Hippo signaling pathway and contributes to cell self-renewal to modulate the pluripotency of embryonic stem cells in various organisms (4). We found that YAP1 mRNA and protein were expressed at a higher level in OSCC tumor specimens and related cell lines. Intriguingly, overexpression of miR-27a-3p in OSCC cell lines could significantly decrease the expression level of YAP1 but also, markedly decreased epithelial to mesenchymal transition (EMT)related factors simultaneously. Epithelial to mesenchymal transition (EMT) is a critical step in the dissemination of malignant diseases (5). In primary tumors, carcinoma cells lose cell-cell adhesion mediated by E-cadherin repression and break through the basement membrane with increased invasive properties. Upregulated expression of EMT-activating transcription factors, including Snail, Twist families and ZEB, could promote tumor invasiveness in xenograft tumor models and cancer cell lines (6). Recently, several studies have shown that miRNAs could regulate invasiveness and metastasis by targeting the transcripts of numerous genes involved in EMT regulation in human cancers (7-9). However, intrinsic 
relationship of OSCC progression related to EMT factors and miRNA regulation still need further research.

In this study, through the gene microarray data analysis and western blot assays, we found miR-27a-3p in OSCC cell lines suppressed its target gene YAP1 expression level and unpredictability decreased the expression level of major EMT-activating transcription factors. Conversely, miR-27a-3p inhibitor transfection reversed this process and promoted cancer invasion by stimulating EMT in OSCC cell lines. Mechanism study showed that miR-27a-3p inhibit EMT in OSCC cell lines and might be involved in the regulation of YAP1-OCT4/Sox 2 signal axis. We report that miR-27a-3p acts as an important upstream regulator related to the EMT via the inhibition of YAP1 in OSCC, which might provide scientific foundation of clinical diagnostics and biomedical research.

\section{Materials and methods}

Cell lines and tissue specimens. Human OSCC cell lines Tca8113, CAL-27, SCC-4, SCC-9, SCC-25, HN-6 and human normal oral keratinocytes (hNOK) cell lines were purchased from the American Type Culture Collection (ATCC). All cell lines were cultured with the complete medium DMEM-F12 medium (Invitrogen) with $10 \%$ heat-inactivated fetal bovine serum (FBS; Gibco), $100 \mathrm{U} / \mathrm{ml}$ of penicillin $\mathrm{G}$ sodium, and $100 \mu \mathrm{g} / \mathrm{ml}$ streptomycin sulfate (Sigma) in a humidified atmosphere containing $5 \% \mathrm{CO}_{2}$ and $37^{\circ} \mathrm{C}$. Fifty paired samples of OSCC tissue and adjacent non-cancerous tissue were obtained from patients with oral squamous cell carcinoma in our department. All the patients provided written informed consent. The histologic diagnosis of tumors was made and agreed upon by two senior pathologists based on World Health Organization (WHO) criteria. This study was approved by the Institutional Review Board and Human Ethics Committee of the Hospital. Tissue samples were flash frozen and stored in liquid nitrogen until used.

Quantitative real-time PCR analysis. Total RNA for quantitative real-time PCR (qRT-PCR) analysis was extracted using 74104 Rneasy Mini kit (Qiagen) and reverse-transcribed into cDNA with the Reverse Transcriptase MMLV (Takara) according to the manufacturer's protocol. Real-time PCR was performed using SYBR Green reagents on the iQ5 Real-Time PCR detection system (both from Bio-Rad). For analysis of miR-27a-3p expression by qRT-PCR, reverse transcription and PCR were carried out using Bulge-Loop miRNA qPCR Primer Set for hsa-miR-143 and U6 snRNA (both from RiboBio, China) according to the manufacturer's instructions. The relative expression level was normalized to that of internal control U6 by using $2^{-\Delta \Delta C t}$ cycle threshold method. Primer sequence miR-27a-3p, 5'-ACACTCCAGCTGGGTTCACAGT GGCTAAG-3'; U6, 5'-CTCGCTTCGGCAGCACA-3'.

miRNA profiling. miRNA expression profiling was performed using total RNA with the Total miRNA qPCR array (System Biosciences, Mountain View, CA, USA). All miRNAs were registered with the Sanger miRBase database. Small nuclear U6 RNA was amplified as normalization control. qPCR was also performed using SYBR-Green as mentioned above. The analysis was performed using software provided by System Biosciences.

Luciferase reporter assay. The 3'-untranslated region (3'UTR) of the human YAP1 gene that was predicted to interact with miR-27a-3p (pMIR-REPORT-YAP1) was synthesized and inserted into pMIR-REPORT (Ambion Inc.). Mutations within potential binding sites were generated by nucleotide replacement of wild-type sequence to inhibit the miR-27a-3p binding. hsa-miR-27a-3p mimic and the negative control were purchased from Ribobio ${ }^{\circledR}$ (miR10000084-1-5). Anti-hsa-miR-27a-3p miScript miRNA inhibitor and miScript inhibitor negative control purchased from Qiagen (MIN0000084). Tca8113 cells were plated into 96 -well plates and were co-transfected with $0.4 \mathrm{mg}$ of the reporter construct, $0.2 \mathrm{mg}$ of control vector, and miR-27a-3p negative controls (NC) after $24 \mathrm{~h}$ incubation. Luciferase values were determined using the Dual-Luciferase reporter assay system.

Cell invasion assay. Cell invasion assays were performed using millicell chambers (Millipore). The chamber was cultivated in $5 \% \mathrm{CO}_{2}$ for $24 \mathrm{~h}$ after the suspension cells $\left(200 \mu \mathrm{l}, 5 \times 10^{4}\right.$ cells $)$ added to the upper chamber. FBS medium (0.5\%) with $10 \%$ FBS $(600 \mu \mathrm{l})$ was added to the lower chamber. Attached cells in the lower section were stained with $0.1 \%$ crystal violet $24 \mathrm{~h}$ later. The invasion rate were quantified by counting the invasion cells in six random fields under a light microscope.

Western blot analysis. Antibodies: YAP1 (CST8579); OCT4 (CST2750); Sox2 (CST3579); and Snail (CST3879) (all from Cell Signaling Technology, USA); Twist (AV37997; Sigma); vimentin (CST5741); E-cadherin (CST5296); and $\beta$-actin (CST4970) (all from Cell Signaling Technology). Cellular lysates were resolved on SDS-PAGE and electrophoretically transferred to polyvinylidene difluoride membranes. Membranes were blocked with a buffer containing Tris (10 mmol/l, pH 7.4), NaCl (150 mmol/l), Tween-20 (0.1\%) and bovine serum albumin $(5 \%)$ and then incubated with the primary antibodies at $4^{\circ} \mathrm{C}$ overnight. Subsequently, washed membranes was treated with appropriate secondary antibodies conjugated to horseradish peroxidase for $1 \mathrm{~h}$. The immunoreactivities were visualized by enhanced chemiluminescence reagents (WBKLS0500; Millipore). $\beta$-actin was used as an internal control.

Statistical analysis. All statistical analyses were performed using SPSS 13.0 software. The data are expressed as mean \pm SD, and one-way ANOVA and an unpaired Student's t-test was used to determine the significant differences of all the results. The level of significance was set at $\mathrm{p}<0.05$.

\section{Results}

miRNA profiling of OSCC tissues and cell lines. miRNAs are recognized as important regulators of post-transcriptional gene expression. In light of miRNA functions, we profiled isolated cells from freshly resected tumors of OSCC patients $(n=5)$ and OSCC cell lines $(n=2$; Tca8113 and CAL-27) using a Total SYBR Green-based qPCR miRNA array (Fig. 1A). Then, we combined the two different data sets in a Venn diagram 
A

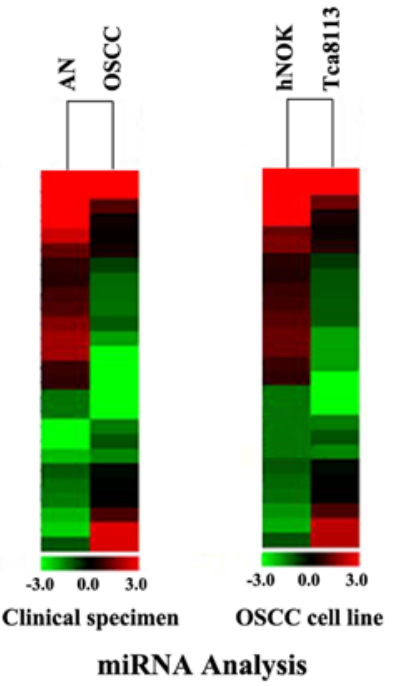

C

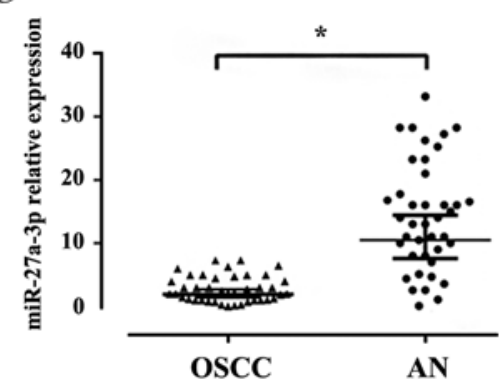

B
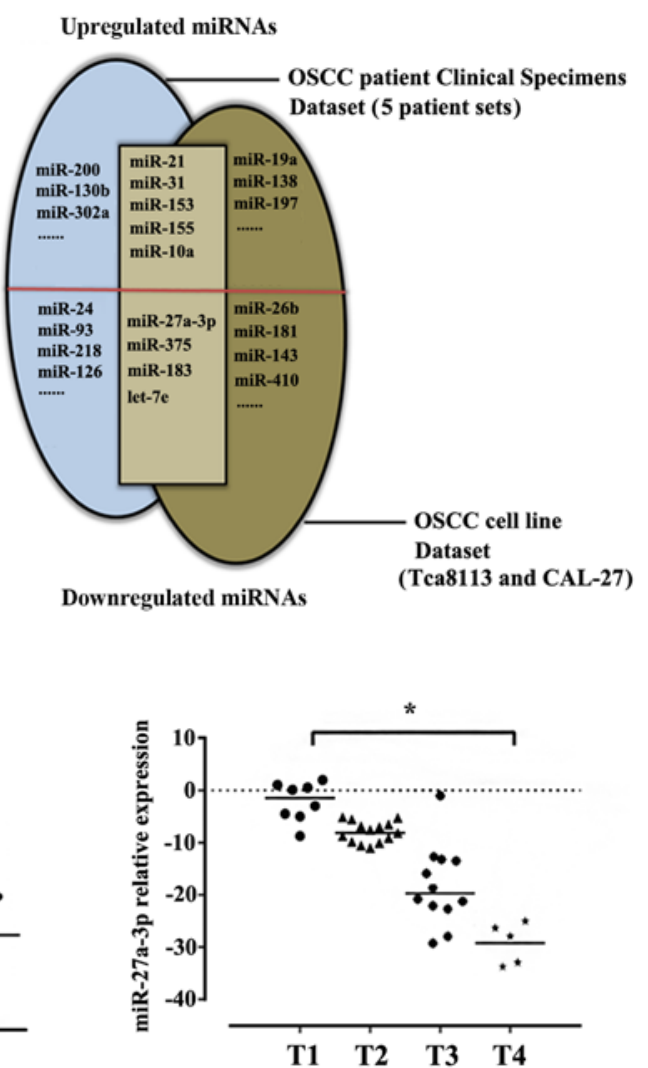

Figure 1. miRNA profiling of OSCC tissues and cell lines. (A) miRNA microarray of deregulated miRNA expression in OSCC paired sample and OSCC cell line (AN, normal adjacent tissues. OSCC, oral squamous cell carcinoma). (B) Analyses of the up and downregulated miRNAs in patient-derived OSCC samples (left; $\mathrm{n}=5$ analyzed tumors) and in OSCC cell lines Tca8113 and CAL-27 (right) are shown. (C) miR-27a-3p expression of fifty pairs of OSCC tissues and adjacent normal tissues from patients were assessed by qRT-PCR analysis (low level expression, 46/50). miR-27a-3p expressions correlated with TNM classification (T1-T4) in OSCC patients were also recorded. The values are standardized to the U6 expression level and represent the mean \pm standard deviation of triplicates. miR-27a-3p also displayed a substantial correlation with $\mathrm{T}$ stage in OSCC patients $\left({ }^{*} \mathrm{P}<0.05,{ }^{* * *} \mathrm{P}<0.01\right)$.

Table I. Dysregulated miRNAs in OSCC patients and OSCC cell lines.

\begin{tabular}{lrrr}
\hline & \multicolumn{3}{c}{ Fold change } \\
\cline { 2 - 4 } miRNAs & (Clinical) & Tca8113 & CAL-27 \\
\hline High expression >3-fold & & & \\
miRNA-21 & 11.13 & 3.53 & 4.03 \\
miRNA-31 & 6.35 & 4.21 & 5.37 \\
miRNA-153 & 6.16 & 3.04 & 3.25 \\
miRNA-155 & 5.53 & 3.48 & 4.13 \\
miRNA-10a & 4.82 & 4.42 & 3.54 \\
Low expression <0.3-fold & & & \\
miRNA-27a-3p & 0.108 & 0.133 & 0.201 \\
miRNA-375 & 0.193 & 0.178 & 0.227 \\
miRNA-183 & 0.236 & 0.288 & 0.112 \\
let-7e & 0.293 & 0.211 & 0.172 \\
\hline
\end{tabular}

and observed expression-altered miRNAs (Fig. 1B). miRNAs that displayed $>3$-fold changes in OSCC patients and common to cell lines were recorded (Table I). Analysis of clinical data and histopathological findings of 50 OSCC samples identified that lower expressions of miR-27a-3p was correlated with poorer outcome of metastatic in OSCC patients. Thus, miR$27 a-3 p$ was selected as the most potential gene involved in the occurrence and development progress of OSCC for further investigation (Fig. 1C).

miR-27a-3p directly targets YAP1 in OSCC cell lines. To investigate the bio-function of miR-27a-3p in OSCC, Tca8113 cell lines transfected with miR-27a-3p mimics were successfully established (Fig. 2A). Then, SYBR Green-Based Gene expression array analysis of cancer related genes was employed to identify the related genes expression changes between the above cell groups (Fig. 2B). Through the data analysis and miRNA target prediction, YAP1 (Yes-associated protein-1) was finally identified as a new potential target of miR27a-3p (Fig. 2C). Results of dual-luciferase reporter assays confirmed that miR-27a-3p directly target YAP1 mRNA 3'UTR region in OSCC cell lines (Fig. 2D). YAP1 is a potent oncogene, which is amplified in various human cancers. An inverse correlation was also observed between the expression levels of miR-27a-3p and YAP1 in both OSCC cell lines and tumor samples (Fig. 2E and F). However, intriguingly, SYBR Green-based gene expression array also showed that overexpression of miR-27a-3p in Tca8113 significantly decreased several epithelial to mesenchymal transition (EMT)-activating 
A

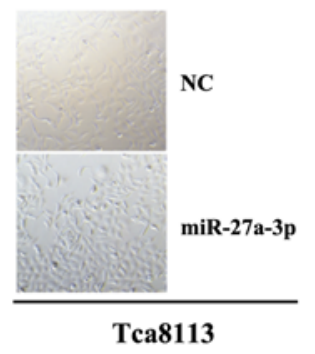

C

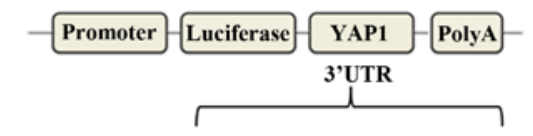

YAP1 3'UTR (WT) 5'...CUAAAUCUGUGAAG... miR-27a-3p 3'...AUCGGUGACACUUU... YAP1 3'UTR (MT) 5'...CUAAAUGCAAUUCG..

E

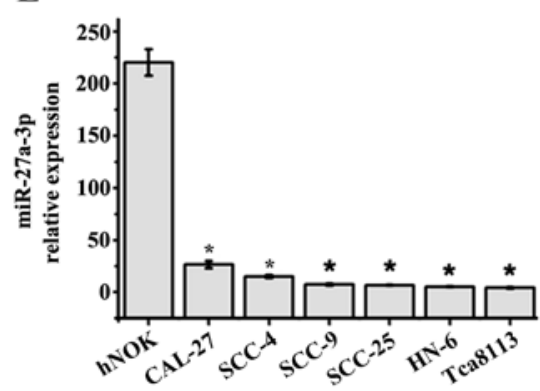

$\mathbf{F}$

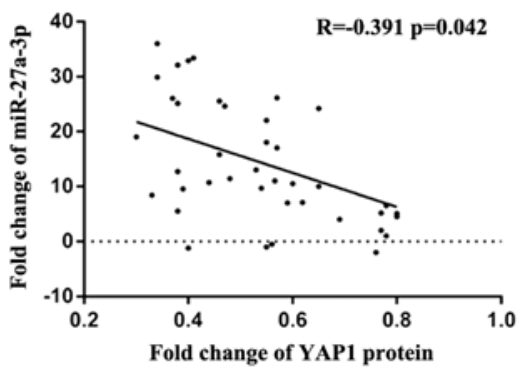

B

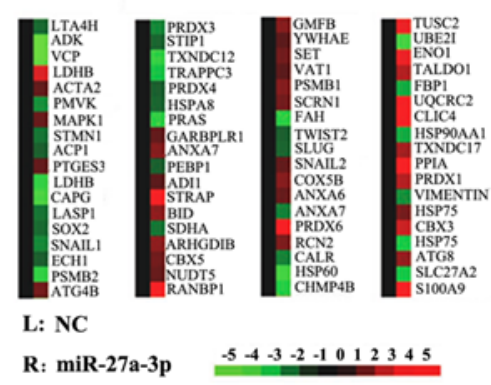

D

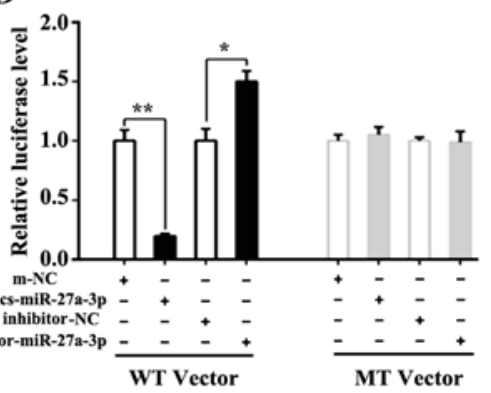

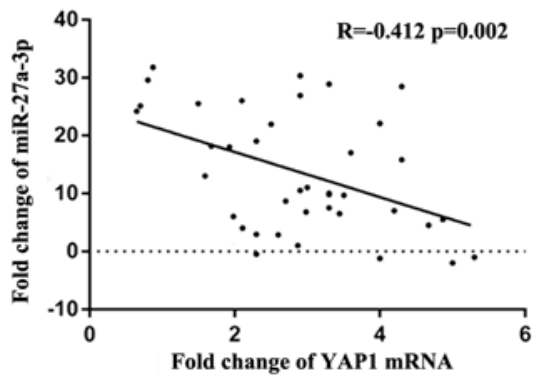

Figure 2. miR-27a-3p directly targets YAP1 in OSCC cell lines. (A) Tca8113 cells transfected with miR-27a-3p mimics (herein after referred to as miR-27a-3p) or negative control (NC) was established and the relative expression of miR-27a-3p was detected by qRT-PCR in $24 \mathrm{~h}$ ("P $<<0.01$ ). (B) SYBR Green-Based Gene expression array and related gene expression changes between the above two groups. Data in gene chip were analyzed by statistical software SPSS15.0 (data not shown). (C) miR-27a-3p/YAP1 alignment by miRanda analysis and the schematic diagram of the pMIR-YAP1/pMIR-YAP1 $1^{\text {mut }}$ paired sequences for miR-27a-3p. (D) Dual normalized luciferase activity of pMIR-YAP1/pMIR-YAP1 ${ }^{\text {mut }}$ reporter in Tca8113 cells transfected with miR-27a-3p (mimics), negative control mimics (NC) or miR-27a-3p inhibitor (inhibitor) or inhibitor negative control (i-NC). All transfection experiments were performed in triplicate and reproduced three times. (E) miR-27a-3p relative expression in OSCC cells lines and YAP1 mRNA relative expression in OSCC cells lines. (F) Pearson's correlation scatter plot of the fold change of miR-27a-3p and YAP1 protein/mRNA.

transcription factors, including Sox2, vimentin (10), Snail (11) and Twist (12). Therefore, Further studies were needed to reveal the regulatory network between miR-27a-3p and EMT in human OSCC cells.

miR-27a-3p inhibits EMT-activating transcription factors through the YAP1-OCT4-Sox2 signal axis. Recent studies have shown YAP1 could regulate OCT4 activity and Sox2 expression to facilitate self-renewal in lung cancer stem-like cells (13) and Sox 2 promoted tumor metastasis by stimulating epithelial-to-mesenchymal transition (EMT)-transcription factors in various tumor tissues (14), which provide some clues as to how miR-27a-3p acts on EMT process in OSCC cells. To verify the interaction between YAP1 and OCT4 in Tca8113, GST-pull down assay was used in this study. Results confirmed that YAP1 could bind to OCT4 directly in Tca8113 cells (Fig. 3A). Then, we detected the mRNA expression level of YAP1 and OCT4 by the real-time qPCR in the presence or 
A

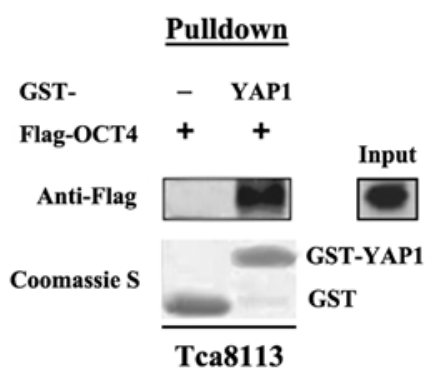

C

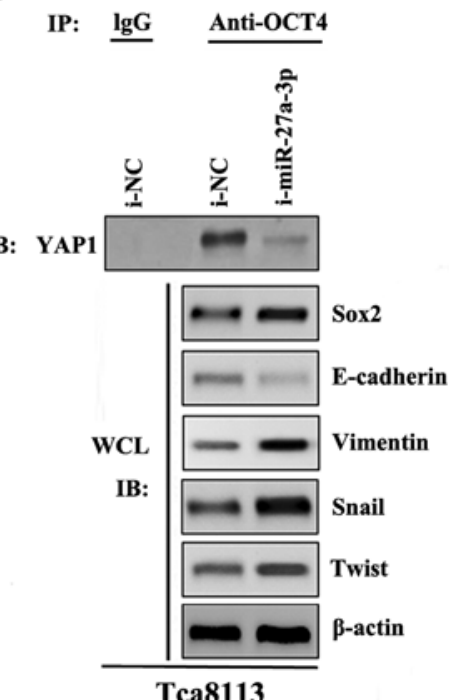

E $\quad$ i-NC i-miR-27a-3p

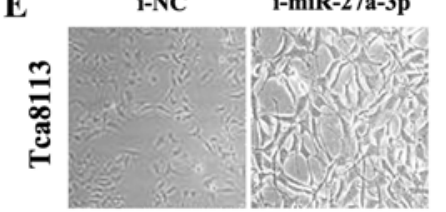

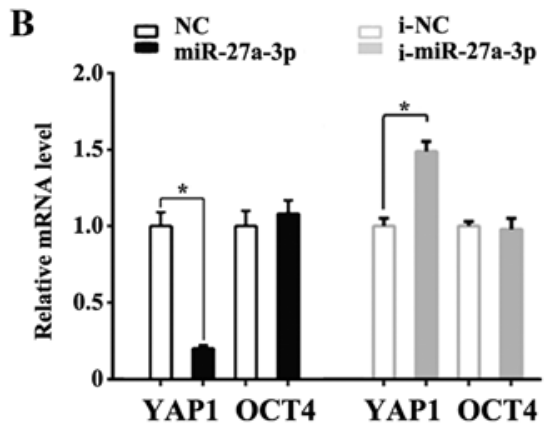

D
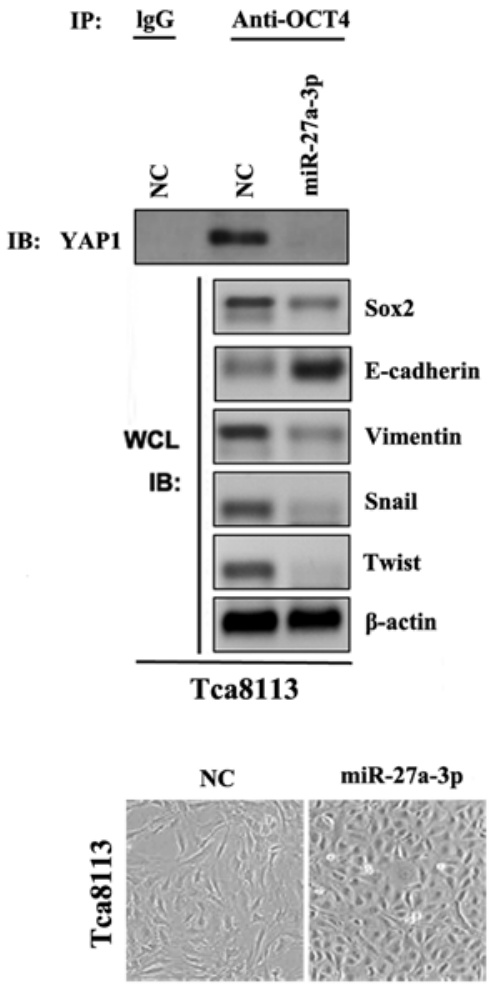

Figure 3. miR-27a-3p inhibits EMT-activating transcription factors through the YAP1-OCT4-Sox2 signal axis. (A) Relative mRNA level of YAP1 and OCT4 in Tca8113 cell line was detected by qRT-PCR after transfected with miR-27a-3p mimics or miR-27a-3p inhibitor in 24 h. (Anti-hsa-miR-27a-3p miScript miRNA inhibitor and miScript inhibitor negative control, MIN0000084; Qiagen). (B) YAP1-OCT4 interaction in vivo in Tca8113. (C) YAP1-OCT4 interaction under the transfection with miR-27a-3p inhibitor or inhibitor negative control (i-NC) in $24 \mathrm{~h}$. (D) YAP1-OCT4 interaction under the transfection with miR-27a-3p or negative control (NC) in $24 \mathrm{~h}$. WCL, whole-cell lysate. IB, immunoblotting; IP, immunoprecipitation. (E) miR-27a-3p transfection induced morphological changes from an extended, fibroblast-like morphology to more epithelial-like cells in $24 \mathrm{~h}$.

absence of miR-27a-3p or miR-27a-3p inhibitor transfection. As the results show in Fig. 3B, miR-27a-3p decreased the YAP1 mRNA level significantly, while the transfection of miR27a-3p inhibitor derepressed YAP1 mRNA level reversely. The OCT4 expression level was not affected in either treatment group. Subsequently, co-immunoprecipitation and western blot assays were both employed to reveal the inner relationship of miR-27a-3p, YAP1 and transcription factors of EMT. In the EMT process, loss of the epithelial marker E-cadherin, and increased expression of mesenchymal markers vimentin are regarded as markers of EMT activation. Additionally, Snail1/2 and Twist $1 / 2$ are also the main upstream inducers of EMT. As shown in Fig. 3C and D, miR-27a-3p was able to decrease the YAP1 expression and influence EMT-related inducers Snail and Twist. miR-27a-3p transfection also induced morphological changes from an extended, fibroblast-like morphology to more epithelial-like cells in Tca8113 (Fig. 3E). Taken together, our findings demonstrated miR-27a-3p acted as one of the vital upstream molecules that mediate the EMT via interruption of YAP1-OCT4/Sox 2 signal axis through targeting YAP1 in OSCC cells.

miR-27a-3p transfection effectively decreases the invasive ability of OSCC cell lines. Description of small aggregates of tumor cells extending from the tumor mass into the adjacent stroma provided morphological evidence of EMT at invasive fronts of human tumors (15). Through prior experimental results and data analysis, we identified miR-27a-3p as one of vital upstream molecules that mediate the EMT in OSCC cell lines. Cell matrix adhesion assay was used to explore the cell invasive ability in the presence or absence of miR-27a-3p transfection in the OSCC cell lines Tca8113 and CAL-27. As 

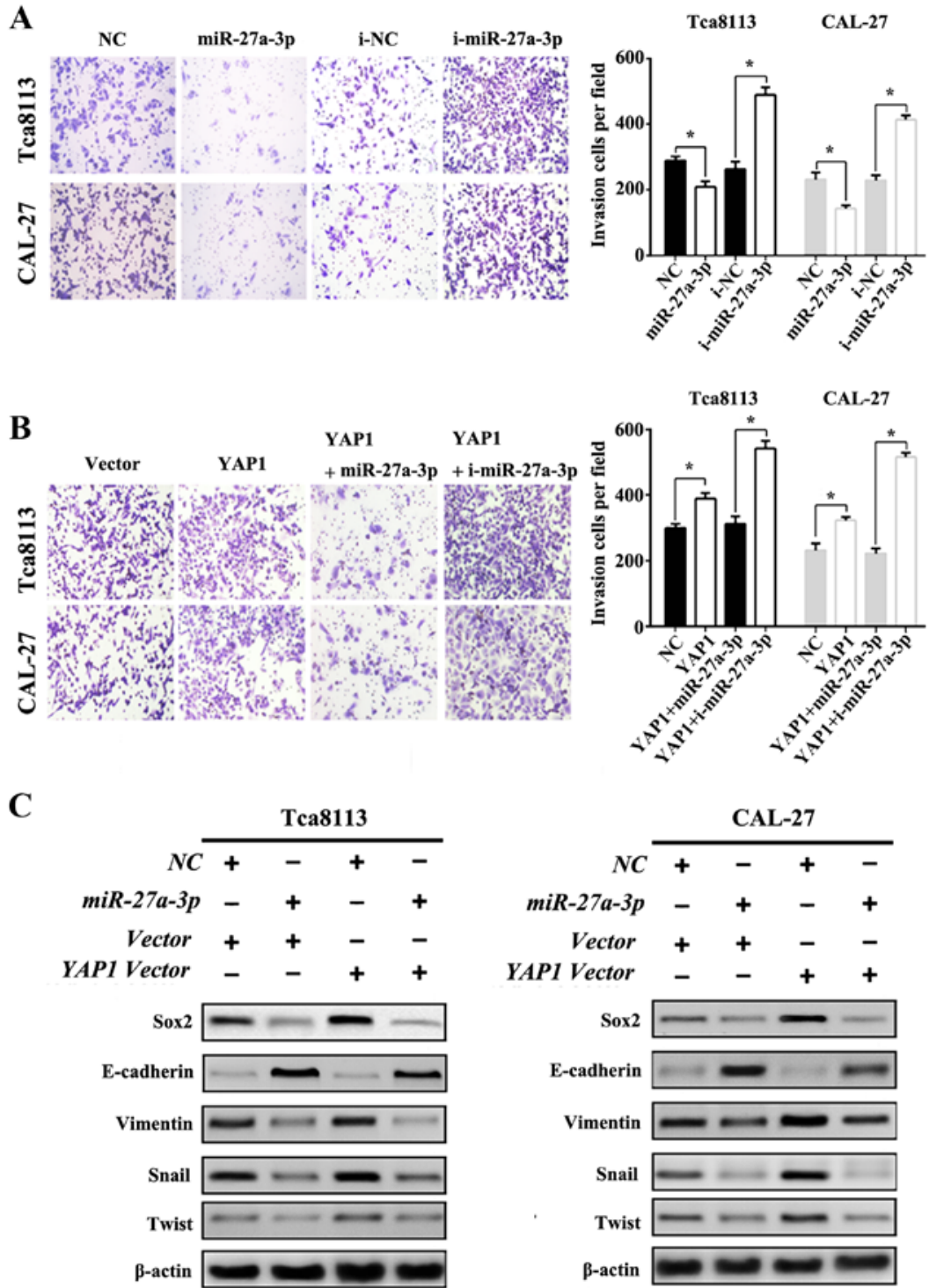

Figure 4. miR-27a-3p transfection effectively decreases the invasive ability of OSCC cell lines. (A) Cell invasion assay by Transwell methods was used to explore the potential function of miR-27a-3p on cell invasiveness in Tca8113 after cells were transfected with miR-27a-3p mimics or miR-27a-3p inhibitor in $24 \mathrm{~h}$. (B) Cell invasion assay by Transwell methods was used to explore cell invasiveness in Tca8113 after cells were transfected with miR-27a-3p or YAP1 vector (PVAX-YAP1 recombinant plasmid) in $24 \mathrm{~h}$. Data are shown as the mean $\pm \mathrm{SD}$ and are representative of three independent experiments. ("P<0.05). (C) Western blot analysis of Sox2, vimentin, Snail and Twist expression from lysates of Tca8113 cells treated with miR-27a-3p or YAP1 for $24 \mathrm{~h}$. $\beta$-actin was used as the internal control.

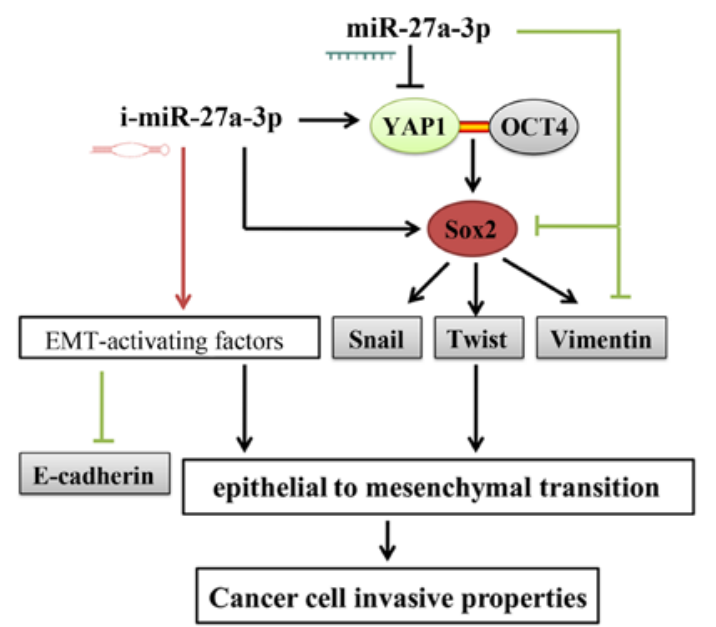

Figure 5. MicroRNA-27a-3p regulates epithelial to mesenchymal transition via targeting YAP1 in oral squamous cell carcinoma cells. shown in Fig. 4A, miR-27a-3p decreased the invasive ability of OSCC cell lines significantly, in contrast, the presence of miR-27a-3p inhibitor reversed miR-27a-3p-induced inhibition of cell invasive ability. Furthermore, recombinant plasmid pVAX-YAP1 (referred as YAP1 vector) was constructed and used in the subsequent experiments. As shown in the Fig. 4B, increased expression of YAP1 could promote the invasive ability of OSCC cell lines. However, miR-27a-3p expression inhibited the invasive effect of YAP1 while miR-27a-3p inhibitor enhanced the invasive effect of YAP1 significantly. Then, western blot assays also confirmed that miR-27a-3p could decrease the expression level of EMT-transcription factors in OSCC cell lines Tca8113 and CAL-27 (as shown in Fig. 4C), suggesting that miR-27a-3p might play pivotal roles in effectively manipulating invasion through the inhibition of EMT in OSCC cells. To better understand the miR-27a-3p function 
related to the EMT process, graphical illustration of this study is shown in Fig. 5.

\section{Discussion}

MicroRNAs (miRNAs) are endogenous small non-coding RNAs that play crucial roles in numerous biological processes (16-18). However, the role of miRNAs in OSCC has not been fully determined. In this study, we profiled isolated cells from freshly resected tumors from OSCC patients and OSCC cell lines using a SYBR-Green-based qPCR miRNA array to identify the expression change of miRNAs. Based on the databases and clinical analysis related to the clincopathological factors in 50 OSCC cases, miR-27a-3p was identified as a potential oncogene in OSCC. Furthermore, SYBR-Green-based gene expression array analysis was employed to identify the related gene expression changes after Tca8113 transfected with miR-27a-3p mimics or the miR-27a-3p negative control. Intriguingly, the status of expression of several epithelial to mesenchymal transition (EMT)-activating transcription factors, including Sox 2, Snail and Twist, were significantly decreased in the miR-27a-3p group when compared with the $\mathrm{NC}$ group.

The epithelial-mesenchymal transition (EMT) is a process by which epithelial cells lose their cell polarity and cell-cell adhesion, which have been regarded as a key developmental program that is often activated during the initiation of metastasis for cancer progression. Many oncogenes have been reported to be involved in tumor growth and metastasis by inducing EMT in various cancers, including OSCC (19). Preliminary data indicate that the main EMT drivers, such as Snail and Twist, could repress the expression of the miR-200 family, but whether miRNAs can also control their expression still need further investigation $(20,21)$. In our study, we identified that YAP1 (Yes-associated protein-1) was the direct target gene of miR-27a-3p through dual-luciferase reporter assay. YAP1 is a oncogenic component of the Hippo signaling pathway that has become the new target of antitumor therapy $(22,23)$. Recently, it has already been reported that YAP1 might act as a transcriptional co-activator for Oct4 and the disruption of this interaction could abrogate the induction of Sox 2 (13). Sox 2 is a key upregulated factor in lung squamous cell carcinoma, directing many genes involved in tumor progression. Previous studies also proved that Sox 2 improves invasiveness of breast cancer cells by promoting EMT which is dependent on Twist 1 and the status of Sox 2 transcription activity (24). To verify the interaction between miR-27a-3p and EMT process, GST-pull down in combination with co-immunoprecipitation and western blot assays were employed to verify our hypothesis. As expected, miR-27a-3p could decrease the YAP1 expression level and EMT-activating transcription factors, which might attribute to the interruption of YAP1-OCT4/Sox 2 signal axis.

By prior experimental results and analysis, we identify miR-27a-3p as one of vital upstream molecules that could mediate the EMT-activating transcription factors through directly target 3'UTR region of YAP1 in OSCC cell lines. With the presence of miR-27a-3p transfection, YAP1 expression was inhibited and induction of Sox 2 was also abrogated since the interaction of YAP1-OCT4 was disrupted. In conclusion, this study supports a possible new mechanism that miR-27a-3p could suppress tumor progression by inhibiting EMT through the YAP1-OCT4/Sox 2 signal axis. Our results might provide scientific foundation of clinical diagnostics and biomedical research on miR-27a-3p and EMT factors in human cancers.

\section{Acknowledgements}

pVAX-YAP1 and the blank vector pVAX plasmid were gifts from Professor Wu Shi (Tsinghua University).

\section{References}

1. Leusink FK, van Es RJ, de Bree R, Baatenburg de Jong RJ, van Hooff SR, Holstege FC, Slootweg PJ, Brakenhoff RH and Takes RP: Novel diagnostic modalities for assessment of the clinically node-negative neck in oral squamous-cell carcinoma. Lancet Oncol 13: e554-e561, 2012.

2. Benfey PN: Molecular biology: microRNA is here to stay. Nature 425: 244-245, 2003.

3. Park K and Kim KB: miRTar Hunter: A prediction system for identifying human microRNA target sites. Mol Cells 35: 195-201, 2013.

4. Sudol M: YAP1 oncogene and its eight isoforms. Oncogene 32: 3922, 2013.

5. Sleeman JP and Thiery JP: SnapShot: The epithelial-mesenchymal transition. Cell 145: 162.e1, 2011.

6. Sánchez-Tilló E, Liu Y, de Barrios O, Siles L, Fanlo L, Cuatrecasas M, Darling DS, Dean DC, Castells A and Postigo A: EMT-activating transcription factors in cancer: Beyond EMT and tumor invasiveness. Cell Mol Life Sci 69: 3429-3456, 2012.

7. Li J, Wang Q, Wen R, Liang J, Zhong X, Yang W, Su D and Tang J: miR-138 inhibits cell proliferation and reverses epithelial-mesenchymal transition in non-small cell lung cancer cells by targeting GIT1 and SEMA4C. J Cell Mol Med 19: 2793-2805, 2015.

8. Yang L, Yang J, Li J, Shen X, Le Y, Zhou C, Wang S, Zhang S, Xu D and Gong Z: MircoRNA-33a inhibits epithelial-to-mesenchymal transition and metastasis and could be a prognostic marker in non-small cell lung cancer. Sci Rep 5: 13677, 2015.

9. Qu MH, Han C, Srivastava AK, Cui T, Zou N, Gao ZQ and Wang QE: miR-93 promotes TGF- $\beta$-induced epithelialto-mesenchymal transition through downregulation of NEDD4L in lung cancer cells. Tumour Biol: Nov 18, 2015 (Epub ahead of print).

10. Vuoriluoto K, Haugen H, Kiviluoto S, Mpindi JP, Nevo J, Gjerdrum C, Tiron C, Lorens JB and Ivaska J: Vimentin regulates EMT induction by Slug and oncogenic H-Ras and migration by governing Axl expression in breast cancer. Oncogene 30: 1436-1448, 2011

11. Naber HP, Drabsch Y, Snaar-Jagalska BE, ten Dijke P and van Laar T: Snail and Slug, key regulators of TGF- $\beta$-induced EMT, are sufficient for the induction of single-cell invasion. Biochem Biophys Res Commun 435: 58-63, 2013.

12. Smit MA and Peeper DS: Deregulating EMT and senescence: Double impact by a single twist. Cancer Cell 14: 5-7, 2008.

13. Bora-Singhal N, Nguyen J, Schaal C, Perumal D, Singh S, Coppola D and Chellappan S: YAP1 regulates OCT4 activity and SOX2 expression to facilitate self-renewal and vascular mimicry of stem-like cells. Stem Cells 33: 1705-1718, 2015.

14. Li X, Xu Y, Chen Y, Chen S, Jia X, Sun T, Liu Y, Li X, Xiang R and Li N: SOX2 promotes tumor metastasis by stimulating epithelial-to-mesenchymal transition via regulation of WNT/ $\beta$-catenin signal network. Cancer Lett 336: 379-389, 2013.

15. Choi HJ, Park KJ, Shin JS, Roh MS, Kwon HC and Lee HS: Tumor budding as a prognostic marker in stage-III rectal carcinoma. Int J Colorectal Dis 22: 863-868, 2007.

16. Xu P, Li Y, Yang S, Yang H, Tang J and Li M: Micro-ribonucleic acid 143 (miR-143) inhibits oral squamous cell carcinoma (OSCC) cell migration and invasion by downregulation of phospho-c-Met through targeting CD44 v3. Oral Surg Oral Med Oral Pathol Oral Radiol 120: 43-51, 2015.

17. Zhang HP, Wang YH, Cao CJ, Yang XM, Ma SC, Han XB, Yang XL, Yang AN, Tian J, Xu H, et al: A regulatory circuit involving miR-143 and DNMT3a mediates vascular smooth muscle cell proliferation induced by homocysteine. Mol Med Rep 13: 483-490, 2016. 
18. Zhang X, Zheng L, Sun Y, Wang T and Wang B: Tangeretin enhances radiosensitivity and inhibits the radiation-induced epithelial-mesenchymal transition of gastric cancer cells. Oncol Rep 34: 302-310, 2015.

19. Chaw SY, Majeed AA, Dalley AJ, Chan A, Stein S and Farah CS: Epithelial to mesenchymal transition (EMT) biomarkers E-cadherin, beta-catenin, APC and vimentin - in oral squamous cell carcinogenesis and transformation. Oral Oncol 48: 997-1006, 2012.

20. Gill JG, Langer EM, Lindsley RC, Cai M, Murphy TL, Kyba M and Murphy KM: Snail and the microRNA-200 family act in opposition to regulate epithelial-to-mesenchymal transition and germ layer fate restriction in differentiating ESCs. Stem Cells 29: 764-776, 2011.

21. Kong D, Li Y, Wang Z, Banerjee S, Ahmad A, Kim HR and Sarkar FH: miR-200 regulates PDGF-D-mediated epithelial-mesenchymal transition, adhesion, and invasion of prostate cancer cells. Stem Cells 27: 1712-1721, 2009.
22. Keren-Paz A, Emmanuel R and Samuels Y: YAP and the drug resistance highway. Nat Genet 47: 193-194, 2015.

23. Cottini F, Hideshima T, Xu C, Sattler M, Dori M, Agnelli L, ten Hacken E, Bertilaccio MT, Antonini E, Neri A, et al: Rescue of Hippo coactivator YAP1 triggers DNA damageinduced apoptosis in hematological cancers. Nat Med 20: 599-606, 2014.

24. Wu F, Ye X, Wang P, Jung K, Wu C, Douglas D, Kneteman N, Bigras G, Ma Y and Lai R: Sox2 suppresses the invasiveness of breast cancer cells via a mechanism that is dependent on Twist1 and the status of Sox 2 transcription activity. BMC Cancer 13: 317, 2013. 